\title{
Las fortalezas en personas mayores como factor que aumenta el bienestar
}

\author{
Encarnación Ramírez, Ana R. Ortega y Rafael Martos \\ Universidad de Jaén (España)
}

\begin{abstract}
En las últimas décadas se ha incrementado el estudio de las fortalezas psicológicas como factor que afecta al bienestar. Éstas pueden ser consideradas como un subconjunto de rasgos de personalidad a los que se otorga un valor moral, definiéndose como cualidades naturales que las personas están intrínsecamente motivada a utilizar porque aumentan la calidad de vida. Las investigaciones demuestran que actúan como factores de protección y prevención de la psicopatología y de los problemas de conducta en las diferentes etapas evolutivas. Hay fortalezas que son más relevantes en personas mayores y su entrenamiento estaría dirigido a influir sobre el comportamiento y el funcionamiento emocional con el objetivo de aumentar la satisfacción con la vida. En el presente trabajo se analizan las fortalezas que predominan en adultos mayores y se propone un programa de intervención para potenciarlas con la finalidad de mejorar su salud y calidad de vida. Se trata de una propuesta novedosa ya que, hasta el momento, las intervenciones en este ámbito han ido encaminadas a paliar diferentes problemas tanto de manera aislada como complemento de terapias de tipo más tradicional pero no como prevención de la aparición de trastornos habituales en edades avanzadas.
\end{abstract}

Palabras clave: Fortalezas, psicología positiva, bienestar, calidad de vida.

Strengths in the elderly as a factor that increases wellbeing. In the last decades there has been increased the study of the psychological strengths as factor that concerns the wellbeing. They are considered as a subset of features of personality to which a moral value is added, being defined as natural qualities that the persons are intrinsically motivated to use as they increase the quality of life. The investigations demonstrate that they act as factors of protection and prevention of the psychopathology and of the problems of behavior in the different stages of life. There are strengths that are more relevant in older people and their training would try to influence the behavior and the emotional functioning to increase the satisfaction with the life. In the present work we analyze the strengths that predominate in older and we propose a program of intervention to promote the improve their health and quality of life. This is a new proposal because, until now, interventions in this area have been aimed at palliate various problems or as a complement to traditional therapy but not to prevent the onset of common disorders in the elderly.

Keywords: Strengths, positive psychology, wellbeing, life quality.

Correspondencia: Encarnación Ramírez Fernández. Universidad de Jaén. Paraje de las Lagunillas, s/n, 23071. Jaén (España). E-mail: eramirez@ujaen.es 
Las fortalezas son características positivas de la personalidad que pueden actuar dirigiendo $\mathrm{u}$ organizando nuestro propio comportamiento. Actúan como características fijas que se repiten a lo largo del tiempo y en diferentes situaciones y se desarrollan o se modifican a partir de las interacciones del individuo con el propio entorno.

Las fortalezas y virtudes psicológicas están teniendo cada vez una mayor importancia en psicología, ya que se ha comprobado en diferentes investigaciones que pueden actuar como amortiguadores y barreras frente al trastorno mental, además de contribuir a mejorar el bienestar y la salud (Wood, Linley, Maltby, Hashdan, y Hurling, 2011). Se ha demostrado que las fortalezas presentan correlaciones positivas con satisfacción con la vida, autoestima y afecto positivo y negativas con variables psicopatológicas como la depresión o el estrés (Giménez, Vázquez, y Hervás, 2010). Por tanto, su potenciación puede promover el bienestar, mejorando la calidad de vida de las personas.

En las últimas décadas se ha observado un incremento en el estudio de las fortalezas psicológicas como factor que afecta al bienestar (Biswas-Diener, Kashdan, y Minhas, 2011; Hart y Sasso, 2011). Así, se han relacionado con emociones positivas como alegría, orgullo, satisfacción, amor, compasión y diversión. Güsewell y Ruch (2012) encontraron que las correlaciones más altas entre fortalezas de carácter y emociones positivas se producen entre las fortalezas de entusiasmo y esperanza, y las emociones de alegría y satisfacción.

Puesto que parece existir un efecto positivo de las fortalezas, se han realizado numerosas intervenciones psicológicas con el objetivo de fomentar o entrenar a las personas en diversas fortalezas, como una opción para promover el bienestar y la salud (Geraghty, Wood, y Hyland, 2010a; 2010b). Algunos de los resultados obtenidos en estas intervenciones muestran que se produce un incremento significativo en el estado de ánimo positivo, así como un incremento en la satisfacción con la vida y una reducción de los síntomas en enfermedades y en quejas de salud subjetivas (Remor y Amorós-Gómez, 2012).

Proyer, Rusch, y Buschor (2012), llevaron a cabo un programa con 178 adultos en el cual se entrenaban las principales fortalezas. La muestra se dividió en tres grupos, mientras el primero fue entrenado en las fortalezas de curiosidad, gratitud, optimismo, humor y entusiasmo, el segundo trabajó con las fortalezas de apreciación de la belleza, creatividad, amabilidad, amor por el aprendizaje y esperanza. Por último, el tercero sirvió como control y no recibió entrenamiento en ninguna de las fortalezas. Los resultados indicaron que hubo un aumento significativo de la satisfacción en el primer grupo. Esto podría deberse a que las fortalezas entrenadas en él están ampliamente relacionadas con la satisfacción con la vida. 
Por otra parte, la adquisición y desarrollo de las fortalezas está mediatizado por diversas características personales entre las que cabe destacar la edad de los individuos. Así, Park y Peterson (2006) encontraron que las fortalezas más comunes entre los jóvenes eran la gratitud, el sentido del humor y el amor mientras que las menos frecuentes fueron la prudencia, el perdón, la espiritualidad y la autorregulación. Vázquez y Hervás (2009) encontraron que la esperanza, el trabajo en equipo y la vitalidad son relativamente más comunes entre los jóvenes que entre los adultos, mientras que la apreciación de la belleza, la autenticidad, el liderazgo y la apertura de mente se presentan con más frecuencia entre las personas adultas. Estas diferencias encontradas abren un camino hacia un estudio más profundo acerca de las fortalezas más utilizadas en cada una de las etapas del desarrollo evolutivo de las personas y sus relaciones con la calidad de vida. El presente trabajo se centra en el estudio de las fortalezas que se relacionan, en personas mayores, con la felicidad, la satisfacción con la vida y el afecto positivo.

A partir de la etapa adulta, es importante el reconocimiento de los recursos, capacidades y fortalezas existentes para promover cambios que contribuyan a un mayor bienestar y a mejorar la calidad de vida. Los desafíos que acontecen en este estadio de la vida hacen que los adultos adquieran una serie de mecanismos de defensa. La fortaleza del sentido del humor ha sido uno los principales mecanismos de defensa ante el envejecimiento, ya que permite la expresión de emociones sin malestar y sin efectos desagradables sobre los demás (Ruch, Proyer, y Weber, 2010). Asimismo, Zavarce-Armas (2011) determinó que los adultos parecen valorar más las virtudes de justicia, trascendencia, humanidad y amor aunque estas fortalezas están mediadas por diferentes factores como los culturales o las circunstancias específicas y experiencias de la vida que cada individuo experimente a lo largo de su historia personal.

Un estudio llevado a cabo por Proyer, Gander, Wyss, y Ruch (2011), se centró en cuáles son las fortalezas clave que contribuyen a la satisfacción con la vida en el pasado, presente y futuro. Los resultados indican que curiosidad, esperanza, gratitud, amor y entusiasmo contribuyen a alcanzar la satisfacción en el presente, la apreciación de la belleza es una fortaleza más relacionada con satisfacción en el pasado, mientras que esperanza y espiritualidad fueron los mejores predictores de la satisfacción en la vida futura.

Identificar y ser capaz de desarrollar nuestras fortalezas psicológicas puede producir aumentos en el bienestar individual tal y como muestra el meta-análisis realizado por Bolier et al. (2013), cuyos resultados indican que las intervenciones basadas en psicología positiva mejoran significativamente el bienestar subjetivo y psicológico, al igual que reducen síntomas depresivos.

De acuerdo con lo expresado anteriormente, el objetivo del presente trabajo consistió en identificar aquellas fortalezas que se relacionan, en personas mayores con 
medidas de bienestar, así como establecer si dichas fortalezas son factores predictores del mismo con el fin de proponer una intervención basada en su entrenamiento.

\section{MÉTODO}

\section{Participantes}

La muestra estuvo compuesta por 120 participantes, 68 varones y 52 mujeres, con edades comprendidas entre los 60 y los 89 años $(M=72.4 ; S D=4.32)$, seleccionados de los Centros de Mayores en diferentes municipios (Bailén, Guarromán, Linares y Vilches) de la provincia de Jaén. Su participación fue totalmente voluntaria no recibiendo ningún tipo de compensación por ello.

\section{Instrumentos}

Los cuestionarios que se utilizaron en el estudio fueron los siguientes:

Escala de Satisfacción con la vida (Diener, Emmons, Larsen, y Griffin, 1985; versión castellana de Pons, Atienza, Balaguer, y García-Merita, 2002), compuesta por 5 ítems que evalúan el juicio global que hacen las personas sobre su satisfacción con la vida. Presenta una consistencia interna medida mediante alfa de Cronbach de 0.87.

Escala de felicidad subjetiva (Lyubomisky y Lepper, 1999; adaptación al castellano de Vera-Villarroel, Celis, y Córdova, 2011), es una escala tipo Likert de 4 ítems que mide la felicidad subjetiva global mediante enunciados en los que la persona se evalúa a si misma o bien se compara con quien la rodea. Tiene una alfa de Cronbach de 0.86 .

Escala de Afectividad positiva y negativa (PANAS; Watson, Clark, y Tellegen, 1988, adaptación al castellano de Sandín et al., 1999). La escala mide 2 factores (afecto positivo y afecto negativo) y utiliza para medir cada factor 10 ítems. La consistencia interna para el afecto positivo oscila entre 0.86 a 0.90 ; y para el afecto negativo de 0.84 a 0.87 .

Cuestionario de Fortalezas (VIA; Peterson y Seligman, 2004), compuesto por 48 ítems cuyas opciones de respuesta oscilan de "muy propio de mí" a "impropio de mí". Mide el grado en el que una persona posee cada una de las 24 fortalezas. Presenta un coeficiente alfa de Cronbach que oscila entre 0.71 y 0.87 .

\section{Procedimiento}

La participación en el estudio se solicitó a través de distintos centros públicos donde se llevan a cabo talleres de pintura, lectura, cultura general y estimulación cognitiva en los municipios de Bailén, Guarromán, Linares y Vilches. A los directores de dichos centros se les informó de las características del estudio así como de la posibilidad de participar en éste. Una vez obtenida la autorización del centro, se convocó a todos sus 
socios a una reunión en la que se dio la información referente al estudio. Tras la obtención del consentimiento informado, se procedió a la administración de los distintos cuestionarios en el propio centro.

\section{Análisis de datos}

Se llevó a cabo un estudio correlacional de corte transversal con el fin de estudiar las relaciones existentes entre las diferentes variables medidas mediante los instrumentos descritos previamente. Una vez obtenidos los datos se calcularon los coeficientes de correlación entre las diferentes variables y se realizaron los análisis de regresión pertinentes mediante el método de pasos sucesivos. Las decisiones estadísticas se tomaron a un nivel de .05 .

\section{RESULTADOS}

En primer lugar se procedió a calcular la correlación entre las diferentes fortalezas y las medias de bienestar. Los resultados mostraron que existe una correlación significativa entre la Felicidad y las fortalezas de Curiosidad $\left(r_{x y}=.192, p<.05\right)$, Valentía $\left(r_{x y}=.228, p<.05\right)$, Integridad $\left(r_{x y}=.198, p<.05\right)$, Liderazgo $\left(r_{x y}=.228, p<.05\right)$, Autocontrol $\left(r_{x y}=.180, p<.05\right)$ y Perdón $\left(r_{x y}=.193, p<.05\right)$. En el caso del Afecto Positivo, se obtuvieron correlaciones significativas con las fortalezas de Amor por el conocimiento $\left(r_{x y}=.186, \mathrm{p}<.05\right)$, Inteligencia Social $\left(r_{x y}=.268, p<-01\right)$, Esperanza $\left(r_{x y}=.182, p<.05\right)$ y Sentido del humor $(r=.231, p<.05)$. La Satisfacción con la vida presentó una relación significativa con las fortalezas Inteligencia Social $\left(r_{x y}=.208, p<.05\right)$, Perspectiva $\left(r_{x y}=-.189, p<.05\right)$, Amor $\left(r_{x y}=.414, p<.01\right)$, Liderazgo $\left(r_{x y}=.229, p<.05\right)$, Disfrute de la belleza $\left(r_{x y}=.277, p<.01\right)$, Gratitud $\left(r_{x y}=.191, p<.05\right)$, Perdón $\left(r_{x y}=.241, p<.01\right) \mathrm{y}$ Entusiasmo $\left(r_{x y}=.192, p<.05\right)$.

Con el fin de conocer hasta qué punto estas fortalezas pueden ser buenos predictores de las medidas de bienestar, se realizó un análisis de regresión por pasos sucesivos para cada una de estas medidas. Este análisis mostró que el mejor modelo para predecir la Felicidad contiene como predictores las fortalezas de Liderazgo y Valentía $\left(R_{\text {corregida }}^{2}=.079, F_{(2.117)}=6.97, p=.003\right)$.

Por otra parte, en el caso del Afecto Positivo, la única fortaleza que parece predecirlo es la fortaleza Inteligencia Social. Este modelo presenta una $R_{\text {corregida }}^{2}=.064$, $F_{(1.118)}=9.10, p=.003$.

El análisis de regresión realizado utilizando la Satisfacción con la vida como variable dependiente mostró que el mejor modelo para predecir esta variable es el formado por las fortalezas Amor, Entusiasmo, Disfrute de la belleza y Gratitud $\left(R_{\text {corregida }}^{2}=.288, F_{(4.115)}=13.026, p=.000\right)$. 


\section{DISCUSIÓN Y CONCLUSIONES}

De acuerdo con los resultados obtenidos en este estudio, en el caso de las personas mayores, no todas las fortalezas tienen una relación significativa con las medidas de bienestar, en concreto, nuestros datos muestran que las fortalezas que correlacionan con estas medidas son Curiosidad, Valentía, Integridad, Liderazgo, Autocontrol, Perdón, Amor, Inteligencia Social, Esperanza, Sentido del humor, Perspectiva, Disfrute de la belleza, Gratitud y Entusiasmo. Por otra parte, la Valentía y el Liderago son buenos predictores de la Felicidad, el Amor, el Entusiasmo, el Disfrute y la Gratitud predicen la Satisfacción con la vida y la Inteligencia Social es un buen predictor del Afecto positivo. Estos resultados son similares a los obtenidos en otros trabajos llevados a cabo con este mismo tipo de población (Güsewell y Rusch, 2012; Ruh, Proyer, y Buschor, 2012) si bien no concuerdan exactamente las mismas fortalezas. Hay suficientes investigaciones que indican que las personas que usan sus fortalezas de una manera más frecuente experimentan un mayor bienestar subjetivo y psicológico, más emociones positivas, mejores estrategias de afrontamiento ante el estrés y relaciones sociales más saludables (DeWall, Lambert, Pond, Kashdan, y Fincham, 2012; Linley y Burns, 2010; Proctor, Maltby, y Linley, 2009). Las discrepancias entre los resultados de diferentes trabajos pueden explicarse, al menos en parte, por el hecho de que no se han utilizado exactamente las mismas medidas de bienestar en todos los estudios y porque el uso de las fortalezas está mediatizado por factores culturales y de la experiencia (Remor, Amorós-Gómez, y Carrobles, 2010).

En la literatura existen diversas investigaciones que muestran como el entrenamiento de las fortalezas incrementa los niveles de satisfacción y felicidad. Así, Ho, Yeung, y Kwok (2014) llevaron a cabo una intervención en Optimismo, Gratitud, Disfrute, Curiosidad, Valentía y Altruismo. Sus resultados muestran que los niveles de bienestar subjetivo aumentaron y se redujeron los niveles de depresión. En esta misma línea, Kaczmarek et al. (2015) encontraron que escribir un diario de Gratitud produce un incremento mayor en el bienestar que otro tipo de entrenamiento en esta misma fortaleza. De acuerdo con los trabajos previos y con nuestros propios resultados, parece interesante realizar una intervención dirigida a las personas mayores en la que se entrenen las fortalezas que mejor predicen el bienestar con el objetivo de mejorarlo y potenciarlo.

Algunas de las fortalezas que, de acuerdo con nuestros datos, deberían trabajarse son ampliamente conocidas en la literatura pero otras como la Valentía, el Amor o la Inteligencia social no han sido muy estudiadas en este contexto. En concreto, nuestra propuesta es llevar a cabo una intervención con cinco bloques dedicados cada uno de ellos a una o varias fortalezas relacionadas. En el primero, se entrenaría la Valentía describiendo en qué consiste esta fortaleza y presentando situaciones difíciles y 
que producen temor con el fin de que los participantes realicen actividades encaminadas a superarlas. Un segundo bloque estaría dedicado a la Inteligencia social, describiéndola dentro de la Inteligencia emocional. En este bloque se trabajaría la empatía, las habilidades sociales y habilidades de comunicación, resolución de conflictos y liderazgo en grupos. El tercer bloque está dedicado al Amor. En este caso se trata de enseñar a querer y dejarse querer por los demás, valorando las relaciones profundas e íntimas que aporten apoyo psicológico. La Gratitud se entrenaría en el cuarto bloque de la intervención, usando para ello tareas conocidas como llevar un diario de Gratitud y rememorar las cosas buenas que han sucedido. Finalmente, el quinto bloque, se dedicaría al disfrute de la vida proponiendo tareas que permitan poner de manifiesto el entusiasmo por las cosas y los acontecimientos, así como aprender a disfrutar de los grandes y los pequeños momentos que depara la vida.

En definitiva, se trata de hacer que nuestros mayores utilicen su potencial para que sigan teniendo una vida plena, llena de satisfacciones consigo mismos y con su entorno. Lograr que mantengan el interés, que se enfrenten a nuevos retos, a pesar de haber entrado en una etapa de su vida que pueden vivir con incertidumbre. Esto, no solo contribuirá a lograr individuos más felices, sino también a que participen en la sociedad de una forma plena, aportando su experiencia vital.

\section{REFERENCIAS}

Biswas-Diener, R., Kashdan, T.B., y Minhas, G. (2011). A dynamic approach to psychological strength development and intervention. Journal of Positive Psychology, 6, 106-118.

Bolier, L., Haverman, M., Westerhof, G.J., Riper, H., Smit, F., y Bohlmeijer, E. (2013). Positive psychology interventions: A meta-analysis of randomized controlled studies. BMC Public Health, 13, 119. doi: http://dx.doi.org/10.1186/1471-2458-13-119.

DeWall, C.N., Lambert, N.M., Pond, R.S. Kashdan, T.B., y Fincham, F.D. (2012). A grateful heart is a nonviolent heart: Cross-sectional, longitudinal, experience sampling, and experimental evidence. Social Psychological and Personality Science, 3, 232-240.

Diener, E., Emmons, R., Larsen, R.J., y Griffin, S. (1985). The satisfaction with life scale. Journal of Personality Assessment, 49, 71-75.

Ferrando, P.J., Chico, E., y Tous, J.M. (2002). Propiedades psicométricas del test de optimismo Life Orientation Test. Psicothema, 14, 673-680.

Geraghty, A.W.A., Wood, A.M., y Hyland, M.E. (2010a). Attrition from self-directed interventions: Investigating the relationship between psychological predictors, intervention content and dropout from a body dissatisfaction intervention. Social Science \& Medicine, $71,30-37$.

Geraghty, A.W.A., Wood, A.M., y Hyland, M.E. (2010b). Dissociating the facets of hope: Agency of pathways predict attrition from unguided self-help in opposite directions. Journal of Research in Personality, 44, 155-158.

Giménez, M., Vázquez, C., y Hervás, G. (2010). El análisis de las fortalezas psicológicas en la adolescencia: Más allá de los modelos de vulnerabilidad. Psychology, Society, y Education, 2, 97-116. 
Gusewell, A., y Ruch, W. (2012). Are only emotional strengths emotional? Character strengths and disposition to positive emotions. Applied Psychology. Health and Well - being, 4(2), 218-239.

Hart, K.E., y Sasso, T. (2011). Mapping the contours of contemporary positive psychology. Canadian Psychology, 52, 82-92.

Ho, H.C.Y., Yeung, D.Y., y Kwok, S.Y.C.L. (2014). Development and evaluation of the positive psychology intervention for older adults. Journal of Positive Psychology, 9, 187-197.

Kaczmarek, L.D., Kashdan, T.B., Drazkowski, D., Enko, J., Kosakowski, M., Szäefer, A., y Bujacz, A. (2015). Why do people prefer gratitude journaling over gratitude letters? The influence of individual differences in motivation and personality on web based interventions. Personality and Individual Differences, 75, 1-6.

Linley, P.A., y Burns, G.W. (2010). Strenghspotting. En G.H. Burns (Ed.) Happiness, healing, enhancement: Your casebook collection for applying positive psychology in therapy (pp. 3-14). Hoboken, NJ: John Wiley and Sons.

Lyubomirsky, S., y Lepper, H.S. (1999). A measure of subjective happiness: Preliminary reliability and construct validation. Social Indicators Research, 46, 137-155.

Park, N., y Peterson, C. (2006). Character strengths in organizations. Journal of Organizational Behavior, 27, 1-6.

Peterson, C., y Seligman, M.E.P. (2004). Character strenghts and virtues: A handbook and classification. Washington, D.C.: American Psychological Association.

Pons, D., Atienza, F.L., Balaguer, I., y García-Merita, M.L. (2002). Propiedades psicométricas de la Escala de Satisfacción con la Vida en personas de la tercera edad. Revista Iberoamericana de Diagnóstico Evaluación Psicológica, 13, 71-82.

Proctor, C., Maltby, J., y Linley, P.A. (2009). Strengths use as a predictor of well-being and health-related quality of life. Journal of Happiness Studies, 10, 583-630.

Proyer, R. T., Gander, F., Wyss, T., y Ruch, W. (2011). The relation of character strengths to past, present, and future life satisfaction among German-speaking women. Applied Psychology: Health and Well-being, 3(3), 370-384.

Proyer, R.T., Rusch, W., y Buschor, C. (2012). Testing Strengths-Based Interventions: A Preliminary Study on the Effectiveness of a Program Targeting Curiosity, Gratitude, Hope, Humor, and Zest for Enhancing Life Satisfaction. Journal of Happiness Studies, $14,275-292$.

Remor, E., y Amorós, M. (2012). Efecto de un programa de intervención para la potenciación de las fortalezas y los recursos psicológicos sobre el estado de ánimo, optimismo, quejas de salud subjetivas y la satisfacción con la vida en estudiantes universitarios. Acta Colombiana de Psicología, 15(2), 75-85.

Remor, E., Amorós, M., y Carrobles, J.A. (2010). Eficacia de un programa manualizado de intervención en grupo para la potenciación de las fortalezas y recursos psicológicos. Anales de Psicología, 26, 49-57.

Ruch, W., Proyer, R.T., y Weber, M. (2010). Humor as a character strength among the elderly: Empirical findings on age-related changes and its contribution to satisfaction with life. Zeitschrift Für Gerontologie Und Geriatrie, 43(1), 13-18. doi: http://dx.doi.org/10.1007 /s00391-009-0090-0.

Sandín, B., Chorot, P., Lostao, L., Joiner, T.E., Santed, M., y Valiente, R. (1999). Escalas PANAS de Afecto Positivo y Negativo: validación factorial y convergencia transcultural. Psicothema, 11(1), 37-51.

Scheier, M., Carver, C., y Bridges, M. (1994). Distinguishing optimism from neuroticism (and trait anxiety, self-mastery, and self-estem): A reevaluation of the Life Orientation Test. Journal of Personality and Social Psychology, 67, 1063-1078. 
Vázquez, C., y Hervás, G. (2009). La ciencia del bienestar. Madrid: Alianza Editorial.

Vera-Villarroel, P., Celis, K., y Córdova, N. (2011). Evaluación de la Felicidad: Análisis Psicométrico de la Escala de Felicidad Subjetiva en población chilena. Terapia Psicológica, 29, 127-133.

Watson, D., Clark, L.A., y Tellegen, A. (1988). Development and validation of brief measures of positive and negative affect: The PANAS scales. Journal of Personality and Social Psychology, 54, 1063-1070.

Wood, A.M., Linley, P.A., Maltby, J., Kashdan, T.B., y Hurling, R. (2011). Using personal and psychological strengths leads to increases in well-being over time: A longitudinal study and the development of the strengths use questionnaire. Personality and Individual Differences, 50(1), 15-19. doi: http://dx.doi.org/10.1016/j.paid.2010.08.004.

Zavarce, P. (2013). Bienestar psicológico y fortalezas del carácter en adultos con estilo de apego seguro e inseguro. (Tesis de maestría, Universidad central de Venezuela). Recuperado de: http://saber.ucv.ve/jspui/handle/123456789/3646

Recibido: 22 de abril de 2015

Recepción Modificaciones: 31 de julio de 2015

Aceptado: 4 de agosto de 2015 